\title{
Common genetic variation near the connexin-43 gene is associated with resting heart rate in African Americans: A genome-wide association study of 13,372 participants
}

\author{
R. Deo, MD, MTR ${ }^{1}$, M.A. Nalls, PhD $^{2}$, C.L. Avery, PhD $^{3}$, J.G. Smith, MD ${ }^{4,5}$, D.S. Evans, PhD, \\ MPH $^{6}$, M.F. Keller, BS ${ }^{7,8}$, A.M. Butler, MS ${ }^{9}$, S.G. Buxbaum, PhD ${ }^{10,11}$, G. Li, MS ${ }^{12}$, P. Miguel \\ Quibrera, MS ${ }^{13}$, E.N. Smith, PhD ${ }^{14,15}$, T. Tanaka, PhD ${ }^{16}$, E.L. Akylbekova, MS ${ }^{17}$, A. Alonso, \\ MD, PhD ${ }^{18}$, D.E. Arking, PhD ${ }^{19}$, E.J. Benjamin, MD, ScM ${ }^{20,21,22,23}$, G.S. Berenson, MD ${ }^{24}$, \\ J.C. Bis, PhD $^{25}$, L.Y. Chen, MD, FHRS ${ }^{26}$, W. Chen, MD, PhD $^{27}$, S.R. Cummings, MD $^{28}$, P.T. \\ Ellinor, MD, PhD ${ }^{29,30}$, M.K. Evans, MD ${ }^{31}$, L. Ferrucci, MD, PhD ${ }^{32}$, E.R. Fox, MD, MPH ${ }^{33}$, S.R. \\ Heckbert, MD, PhD ${ }^{34}$, G. Heiss, MD, PhD ${ }^{35}$, W.C. Hsueh, MPH, PhD ${ }^{36}$, K.F. Kerr, PhD $^{37}$, M.C. \\ Limacher, MD ${ }^{38}$, Y. Liu, MD, PhD ${ }^{39}$, S.A. Lubitz, MD, MPH ${ }^{40}$, J.W. Magnani, MD ${ }^{41,42}$, R. \\ Mehra, MD, MS ${ }^{43}$, G.M. Marcus, MD, MAS, FHRS ${ }^{44}$, S.S. Murray, PhD ${ }^{45}$, A.B. Newman, MD, \\ MPH $^{46}$, O. Njajou, PhD, ScD ${ }^{47}$, K.E. North, PhD ${ }^{48,49}$, D.N. Paltoo, MD, MPH ${ }^{50}$, B.M. Psaty, \\ MD, PhD ${ }^{51,52}$, S.S. Redline, MD, MPH ${ }^{53}$, A.P. Reiner, MD, MSc ${ }^{54}$, J.G. Robinson, MD, MPH ${ }^{55}$, \\ J.I. Rotter, MD ${ }^{56}$, T.E. Samdarshi, MD, MPH ${ }^{57}$, R.B. Schnabel, MD, MSc ${ }^{58}$, N.J. Schork, \\ PhD $^{59}$, A.B. Singleton, PhD $^{60}$, D. Siscovick, MD, MPH ${ }^{61}$, E.Z. Soliman, MD, MSc, $\mathbf{M S}^{62}, \mathbf{N}$. \\ Sotoodehnia, MD, MPH ${ }^{63}$, S.R. Srinivasan, $\mathrm{PhD}^{64}$, H.A. Taylor, MD, MPH ${ }^{65}, \mathrm{M}$. Trevisan, \\ MD $^{66}$, Z. Zhang, MD, MPH ${ }^{67}$, A.B. Zonderman, PhD $^{68,69}$, C. Newton-Cheh, MD, MPH $^{70,71,72,}$ \\ and E.A. Whitsel, MD, MPH ${ }^{73}$
}

1Division of Cardiology, Electrophysiology Section, University of Pennsylvania, Philadelphia, Pennsylvania ${ }^{2}$ Laboratory of Neurogenetics, National Institute on Aging, National Institutes of Health, Bethesda, Maryland ${ }^{3}$ Department of Epidemiology, University of North Carolina, Chapel Hill, North Carolina ${ }^{4}$ Department of Cardiology, Faculty of Medicine, Lund University, Lund, Sweden ${ }^{5}$ Program for Medical and Population Genetics, Broad Institute of Harvard and Massachusetts Institute of Technology, Cambridge, Massachusetts ${ }^{6} \mathrm{California}$ Pacific Medical Center Research Institute, San Francisco, California ${ }^{7}$ Laboratory of Neurogenetics, National Institute on Aging, National Institutes of Health, Bethesda, Maryland ${ }^{8}$ Department of Biological Anthropology, Temple University, Philadelphia, Pennsylvania ${ }^{9}$ Department of Epidemiology, Gillings School of Global Public Health, University of North Carolina, Chapel Hill, North Carolina ${ }^{10}$ Jackson Heart Study, Jackson State University, Jackson, Mississippi ${ }^{11}$ Department of Epidemiology and Biostatistics, Jackson State University School of Health Sciences, Jackson, Mississippi ${ }^{12}$ Cardiovascular Health Research Unit, University of Washington, Seattle, Washington ${ }^{13}$ Department of Epidemiology, Gillings School of Global Public Health, University of North Carolina, Chapel Hill, North Carolina ${ }^{14}$ Department of Pediatrics, University of California at San Diego School of Medicine, La Jolla, California ${ }^{15}$ Rady's Children's Hospital, University of California at San Diego School of Medicine, La Jolla, California ${ }^{16}$ Clinical Research Branch, National Institute on Aging, Baltimore, Maryland ${ }^{17}$ Department of Medicine, University of Mississippi Medical Center, Jackson, Mississippi ${ }^{18}$ Division of Epidemiology and Community Health, School of Public Health, University of Minnesota, Minneapolis, Minnesota ${ }^{19}$ McKusick-

(C) 2013 Elsevier Inc. Published by Elsevier Inc. All rights reserved.

Address reprint requests and correspondence: Dr Rajat Deo, Division of Cardiology, Electrophysiology Section, University of Pennsylvania, 3400 Spruce St, 9 Founders Cardiology, Philadelphia, PA 19104. Rajat.Deo@uphs.upenn.edu.

The first 5 authors should be regarded as first authors. 
Nathans Institute of Genetic Medicine, Johns Hopkins University School of Medicine, Baltimore, Maryland ${ }^{20} \mathrm{NHLBl}$ 's Framingham Study, Framingham, Massachusetts ${ }^{21}$ Department of Epidemiology, Boston University School of Public Health, Boston, Massachusetts ${ }^{22}$ Sections of Cardiology and Preventive Medicine, Department of Medicine, Boston University School of Medicine, Boston, Massachusetts ${ }^{23}$ Evans Memorial Whitaker Cardiovascular Institute, Boton University, Boston, Massachusetts ${ }^{24}$ Department of Epidemiology, Tulane University, New Orleans, Louisiana ${ }^{25}$ Cardiovascular Health Research Unit, Department of Medicine, University of Washington, Seattle, Washington ${ }^{26}$ Cardiac Arrhythmia Center, Cardiovascular Division, University of Minnesota Medical School, Minneapolis, Minnesota ${ }^{27}$ Department of Epidemiology, Tulane University, New Orleans, Louisiana ${ }^{28}$ California Pacific Medical Center Research Institute, San Francisco, California ${ }^{29}$ Cardiovascular Research Center, Massachusetts General Hospital and Harvard Medical School, Boston, Massachusetts ${ }^{30}$ Center for Human Genetic Research, Massachusetts General Hospital and Harvard Medical School, Boston, Massachusetts ${ }^{31}$ Health Disparities Research Section, Clinical Research Branch, National Institute on Aging, National Institute of Health, Baltimore, Maryland ${ }^{32}$ Clinical Research Branch, National Institute on Aging, National Institute of Health, Baltimore, Maryland ${ }^{33}$ Department of Medicine, University of Mississippi Medical Center, Jackson, Mississippi ${ }^{34}$ Department of Epidemiology, School of Public Health, University of Washington, Seattle, Washington ${ }^{35}$ Department of Epidemiology, Gillings School of Global Public Health, University of North Carolina, Chapel Hill, North Carolina ${ }^{36}$ Department of Medicine, University of California, San Francisco, California ${ }^{37}$ Department of Biostatistics, School of Public Health, University of Washington, Seattle, Washington ${ }^{38}$ Division of Cardiovascular Medicine, University of Florida College of Medicine, Gainesville, Florida ${ }^{39}$ Department of Epidemiology and Prevention, Division of Public Health Sciences, Wake Forest University, Winston-Salem, North Carolina ${ }^{40}$ Cardiovascular Research Center, Massachusetts General Hospital and Harvard Medical School, Boston, Massachusetts ${ }^{41}$ Section of Cardiovascular Medicine, Boston University School of Medicine, Boston, Massachusetts ${ }^{42}$ National Heart, Lung and Blood Institute's and Boston University's Framingham Heart Study, Framingham, Massachusetts ${ }^{43}$ Department of Medicine, Case Western School of Medicine, Cleveland, Ohio ${ }^{44}$ Division of Cardiology, Electrophysiology Section, University of California, San Francisco, California ${ }^{45}$ Scripps Translational Science Institute and Scripps Research Institute, La Jolla, California ${ }^{46}$ Department of Epidemiology, Graduate School of Public Health, University of Pittsburgh, Pittsburgh, Pennsylvania ${ }^{47}$ Institute of Human Genetics, University of California, San Francisco, California ${ }^{48}$ Department of Epidemiology, Gillings School of Global Public Health, University of North Carolina, Chapel Hill, North Carolina ${ }^{49}$ Carolina Center for Genome Sciences, Chapel Hill, North Carolina ${ }^{50}$ Office of Science Policy, Office of the Director, National Institutes of Health, Bethesda, Maryland ${ }^{51}$ Cardiovascular Health Research Unit, Departments of Medicine, Epidemiology, and Health Services, University of Washington, Seattle, Washington ${ }^{52}$ Group Health Research Institute, Group Health Cooperative, Seattle, Washington ${ }^{53}$ Division of Sleep Medicine, Department of Medicine, Brigham and Women's Hospital, Harvard Medical School, Boston, Massachusetts ${ }^{54}$ Department of Epidemiology, University of Washington School of Public Health, Seattle, Washington ${ }^{55}$ Department of Epidemiology, University of lowa, lowa City, lowa ${ }^{56}$ Medical Genetics Institute, Cedars-Sinai Medical Center, Los Angeles, California ${ }^{57}$ University of Mississippi School of Medicine, Jackson, Mississippi ${ }^{58}$ Department of General and Interventional Cardiology, University Heart Center, Hamburg-Eppendorf, Germany ${ }^{59}$ Scripps Translational Science Institute and Scripps Research Institute, La Jolla, California ${ }^{60}$ Laboratory of Neurogenetics, National Institute on Aging, Bethesda, Maryland ${ }^{61}$ Cardiovascular Health Research Unit, Departments of Medicine and Epidemiology, University of Washington, Seattle, Washington ${ }^{62}$ Epidemiological Cardiology Research Center (EPICARE), Department of Epidemiology and Prevention, Wake Forest School of Medicine, Winston-Salem, North Carolina ${ }^{63}$ Division of Cardiology, University of Washington, Seattle, Washington ${ }^{64}$ Department of Epidemiology, Tulane University, New Orleans, Louisiana ${ }^{65}$ Departments of Medicine, 
Epidemiology, and Preventive Medicine, University of Mississippi Medical Center, Jackson, Mississippi ${ }^{66}$ Sophie Davis School of Biomedical Education, City College of New York, New York, New York ${ }^{67}$ Epidemiological Cardiology Center (EPICARE), Department of Epidemiology and Prevention, Division of Public Health Sciences, Wake Forest School of Medicine, Winston-Salem, North Carolina ${ }^{68}$ National Institute on Aging, National Institute on Aging, Bethesda, Maryland ${ }^{69} \mathrm{NIH}$-Intramural Research Program, NIH Biomedical Research Center, Baltimore, Maryland 70Program for Medical and Population Genetics, Broad Institute of Harvard and Massachusetts Institute of Technology, Boston, Massachusetts ${ }^{71}$ Cardiovascular Research Center, Massachusetts General Hospital and Harvard Medical School, Boston, Massachusetts ${ }^{72}$ Center for Human Genetic Research, Massachusetts General Hospital and Harvard Medical School, Boston, Massachusetts ${ }^{73}$ Departments of Epidemiology and Medicine, University of North Carolina, Chapel Hill, North Carolina

\section{Abstract}

BACKGROUND—Genome-wide association studies have identified several genetic loci associated with variation in resting heart rate in European and Asian populations. No study has evaluated genetic variants associated with heart rate in African Americans.

OBJECTIVE-To identify novel genetic variants associated with resting heart rate in African Americans.

METHODS-Ten cohort studies participating in the Candidate-gene Association Resource and Continental Origins and Genetic Epidemiology Network consortia performed genome-wide genotyping of single nucleotide polymorphisms (SNPs) and imputed 2,954,965 SNPs using HapMap YRI and CEU panels in 13,372 participants of African ancestry. Each study measured the RR interval (ms) from 10-second resting 12-lead electrocardiograms and estimated RR-SNP associations using covariate-adjusted linear regression. Random-effects meta-analysis was used to combine cohort-specific measures of association and identify genome-wide significant loci $(P \leq$ $\left.2.5 \times 10^{-8}\right)$.

RESULTS-Fourteen SNPs on chromosome 6q22 exceeded the genome-wide significance threshold. The most significant association was for rs9320841 ( $+13 \mathrm{~ms}$ per minor allele; $P=4.98$ $\times 10^{-15}$ ). This SNP was approximately $350 \mathrm{~kb}$ downstream of $G J A 1$, a locus previously identified as harboring SNPs associated with heart rate in Europeans. Adjustment for rs9320841 also attenuated the association between the remaining 13 SNPs in this region and heart rate. In addition, SNPs in $M Y H 6$, which have been identified in European genome-wide association study, were associated with similar changes in the resting heart rate as this population of African Americans.

CONCLUSIONS-An intergenic region downstream of GJA1 (the gene encoding connexin 43, the major protein of the human myocardial gap junction) and an intragenic region within $M Y H 6$ are associated with variation in resting heart rate in African Americans as well as in populations of European and Asian origin.

\section{Keywords}

African Americans; Heart rate; Single nucleotide polymorphisms; Meta-analysis

\section{Introduction}

Multiple studies have found that an elevated resting heart rate is associated with mortality risk $^{1-5}$ including that attributable to sudden cardiac death ${ }^{6}$ and cardiovascular disease. ${ }^{7}$ 
These findings suggest that the function of the sinus node, the dominant pacemaker in the heart, and the autonomic nervous system are associated with adverse clinical outcomes.

Although nongenetic influences of nodal and autonomic function are well known, ${ }^{8}$ genetic factors account for $26 \%-32 \%$ of the variation in resting heart rate in populations of European and Asian ancestry. ${ }^{9-11}$ Genome-wide association studies (GWASs) conducted in populations of European and Asian ancestry have recently identified single nucleotide polymorphisms (SNPs) associated with resting heart rate at several loci including GJA1 on chromosome 6, MYH6 on chromosome 14, CD34 on chromosome 1, and GPR133 on chromosome $12 .{ }^{12-15}$ To the best of our knowledge, however, no study has evaluated the association of genetic variants with heart rate among populations of African descent. Such populations have greater genetic diversity compared to those of European and Asian origin, which may facilitate identification of additional associated loci. ${ }^{16-18}$ It is also unclear whether loci identified in populations of European and Asian ancestry are relevant in populations of African descent.

In an attempt to identify new loci and evaluate existing, known associations, we examined the association of genetic variants with resting heart rate as measured by the RR interval on the electrocardiogram (ECG) among 10 African American cohort studies participating in the Candidate-gene Association Resource (CARe) and the Continental Origins and Genetic Epidemiology Network (COGENT) ECG consortia.

\section{Methods}

\section{Study populations}

The CARe ${ }^{19}$ and COGENT ${ }^{20}$ consortia included 13,372 self-reported African Americans meeting inclusion criteria. The participants originated in 10 cohort studies: the Atherosclerosis Risk in Communities study (ARIC; $n=2391$ ); Baltimore Longitudinal Study of Aging (BLSA; $n=155$ ); Bogalusa Heart Study (BHS; $n=148$ ); Cardiovascular Health Study (CHS; $n=674)$; Cleveland Family Study (CFS; $n=267)$; the Health, Aging, and Body Composition Study (Health ABC; $\mathrm{n}=1054$ ); the Healthy Aging in Neighborhoods of Diversity across the Life Span Study (HANDLS; $\mathrm{n}=945$ ); Jackson Heart Study (JHS; $n=1962)$; Multi-Ethnic Study of Atherosclerosis (MESA; $\mathrm{n}=1627$ ); and Women's Health Initiative clinical trials (WHI; $n=4149$ ). Additional information is provided in the Supplemental Methods, including cohort-specific genotype and imputation quality control methods (see Online Supplements 1 and 2). Participants with missing covariates, poor-quality ECGs, pacemakers or implantable cardioverter-defibrillators, paroxysmal or persistent atrial fibrillation, heart failure, myocardial infarction, second- or third-degree atrioventricular block, and extremes of heart rate ( $>100$ or $<50$ beats $/ \mathrm{min}$ ) were excluded. Participants on medications altering nodal or atrioventricular conduction (betablockers, nondihydropyridine calcium channel blockers, digoxin, type I or III antiarrhythmics) were also excluded.

The study was approved by the institutional review boards at each participating center. Written informed consent was obtained from all participants.

\section{ECG recordings}

A standard 10-second, resting ECG was obtained and recorded digitally on all participants from the 10 cohorts included in this analysis. Standard 12-lead positions were recorded at baseline in all cohort studies using a Marquette MAC PC, MAC6, or MAC1200 ECG machine system (GE Healthcare, Milwaukee, WI). The RR interval (ms) was measured electronically as the unit-corrected inverse of heart rate (beat/min). All ECGs were processed automatically using GE Marquette 12-SL version 2001 running under GE 
Magellan Research Work Station or MC Means. The ECG software is Food and Drug Association approved. Heart rate was calculated from the median RR interval during the 10second recording. Since ECG recordings were simultaneous in all 12 leads, the rate was not affected by the lead from which the RR interval was recorded. The automated nature of calculating heart rate from the median RR interval ensures the highest repeatability with no inter- or intraobserver variability. Poor-quality ECGs were excluded by software algorithms. As an added quality control measure, all ECGs were visually checked.

After a filtering process that results in signal conditioning and averaging, the program generates a median complex. All QRSs of the same shape are aligned in time and the interval measurements depend on the proper identification of fiduciary points, which are determined from an analysis of all 12 leads simultaneously. The intervals are then measured according to published standards. ${ }^{21}$

\section{Genotyping and quality control}

Genome-wide SNP genotyping was performed within each cohort using genotyping arrays from Affymetrix or Illumina (Online Supplement 2). Studies underwent similar quality control procedures (specific details in the Online Supplemental Materials). DNA samples with an array-wide genotyping success rate $<95 \%$ were excluded. Autosomal heterozygosity rates were estimated to identify and exclude samples with poor DNA quality or contamination. Duplicated or contaminated samples were identified from identity by descent estimates and excluded. In addition, SNPs with a genotyping success rate $<90 \%$ per SNP within each cohort, SNPs that map to multiple locations, SNPs where missingness could be predicted from surrounding haplotypes, and SNPs associated with chemistry plates were excluded. African ancestry was confirmed through either principal components ${ }^{22}$ or multidimensional scaling analyses. Population-based (ie, non-family-based) studies used identity-by-descent (IBD) estimates to exclude cryptically related individuals. Subsequent identical SNP filters after imputation and GWAS analyses were applied to summary statistics at the meta-analysis level.

\section{Imputation and quality control}

SNP imputation was performed in each cohort to facilitate the combination of results from different genotyping platforms and to increase genotype coverage. Genotyped SNPs passing quality control metrics described above and reference haplotypes from HapMap Phase 2 (release 22 on NCBI build 36) were used to impute approximately 2.5 million SNPs using MACH v1.16 ${ }^{23}$ or BEAGLE. Untyped SNPs were imputed using a 1:1 ratio of CEU/YRI HapMap reference haplotypes based on consistency across other CARe-COGENT studies. Imputed SNPs were excluded if imputation quality was below 0.30 as reported by $\mathrm{MACH}$ or BEAGLE.

\section{Statistical analysis}

GWAS analysis was performed in either PLINK (ARIC, BHS, CHS, JHS, WHI), R (HANDLS, Health ABC, MESA), ProbA-BEL (WHI), or MERLIN (BLSA) using linear regression with an additive genetic model based on allelic dosages accounting for imputation uncertainty. The family-based CFS study was analyzed using linear mixedeffects models as implemented in the GWAF package for R. ${ }^{24}$ Pedigrees for CFS were confirmed using identity by state or IBD estimates from PREST-Plus (http:// www.utstat.utoronto.ca/sun/Software/Prest/). Previously published analyses indicated that the inclusion of related individuals from the JHS family-based subcohort had little effect on $P$-value inflation. ${ }^{20}$ As a result, these related individuals were included in the present analysis. Eigenvectors were used to adjust for global ancestry in population substructures. Principal components were used to adjust for global ancestry in population stratification. 
Cohort-specific genome-wide association was examined on a SNP-by-SNP basis using simple linear models regressing RR (ms) on allele dosage, age, sex, body mass index, global measures of African ancestry, and, when relevant, study site. Cohort-specific SNP association estimates were combined using fixed- and random-effects meta-analysis, the latter to examine potential effects of among-cohort heterogeneity on the combined estimates and the extent to which it can support qualitative inference to other African American populations. Given evidence of greater genetic and geographical diversity across African American cohorts compared to Europeans and initial evidence of heterogeneity across studies, random-effects estimates, which have wider 95\% confidence intervals than do fixedeffects estimates, were reported in the current meta-analysis. Genomic control methods were applied when study-specific and combined distributions of test statistics suggested early departure from the null $(\lambda>1)$. Genomic inflation factors were evaluated in each cohort before the random-effects meta-analysis and in the combined results. ${ }^{25} \mathrm{We}$ calculated $\mathrm{X}^{2}$ estimates of homogeneity (Cochran's Q) using METAL and $\mathrm{I}^{2}$ estimates with R. Prior to conducting meta-analyses, SNP results with a minor allele frequency $<0.01$ or imputation quality scores $<0.3$ were excluded. In addition, SNPs not seen in $>2$ studies were excluded from the meta-analyses.

To confirm that the random-effects model was not overly conservative, standard fixedeffects meta-analyses were conducted on SNP association estimates for each cohort using METAL (and incorporating genomic control at the meta-analysis level). For the metaanalysis, we prespecified a genome-wide significance threshold of $2.5 \times 10^{-8}$ as suggested for populations of African ancestry, ${ }^{26}$ accounting for approximately 2 million independent common variant tests. Other polymorphisms that were detected at the same locus as the initial SNP were subsequently analyzed in conditional regression models to assess statistical independence. Finally, SNPs that have been identified in prior GWAS but not in the discovery phase of our analysis were evaluated using a less stringent threshold. Specifically, we evaluated 13 genome-wide significant SNPs described by prior RR GWAS in individuals of European and Asian ancestry ${ }^{13-15}$ using a significance level of $3.85 \times 10^{-3}$ (Bonferroni corrected $P$ value calculated as $0.05 / 13)$.

\section{Results}

This GWAS of the RR interval included 13,372 adults of African descent from 10 cohort studies. Each study contributed a widely varying number of participants (range 148-4149). The ARIC, JHS, and WHI studies accounted for the majority of participants in this analysis: $8502(64 \%)$ of 13,372 . On average, the study population was middle-aged (mean 56.5 years; range $35-73$ years) and overweight (mean body mass index $30.8 \mathrm{~kg} / \mathrm{m}^{2}$ ) and $71 \%$ were women.

Genomic inflation was minimal in most studies and modest in the family-based CFS ( $\lambda$ 1.070) and JHS ( $\lambda$ 1.071) (Table 1). Specifically, the lambda estimates from the randomeffects meta-analysis did not suggest inflation of the test statistic (0.868), and the secondary fixed-effects modeling did not show a significant departure from null expectations $(\lambda 1.017)$ (Figures 1A and 1B).

A total of 2,954,965 SNPs were incorporated into this meta-analysis after data quality control. Fourteen SNPs at a largely intergenic region on chromosome 6q22 (Figure 2) reached genome-wide significance. The most significant association at this locus was for rs9320841 ( $+13 \mathrm{~ms}$ per minor allele, standard error $1.7 \mathrm{~ms}$, random effects $P=4.98 \times$ $10^{-15}$ ). This SNP is located in a noncoding region, $350 \mathrm{~kb}$ downstream from GJA1 and 64 $\mathrm{kb}$ upstream from $H M G B 3 P 18$. The magnitude and direction of the association were similar across most cohorts $\left(P_{\text {heterogeneity }}=.45\right)$ as shown in Figure 3. None of the other 13 SNPs in 
this region were independent variants associated with resting heart rate. The results for the regional association plot at the GJA1 locus are depicted in Figure 4. This plot covers 1000 $\mathrm{kb}$ of the genomic region associated with the GJA1 locus and demonstrates strong linkage disequilibrium (LD) with other SNPs in this gene cluster that were associated with variations in heart rate. Adjustment for rs9320841, however, eliminated the significance of these additional SNPs.

We also evaluated a series of SNPs from the chromosome 6q22 locus that were identified in prior European and Asian GWAS. Both rs9398652 and rs12110693 in the 6q22 locus were associated with the RR interval, which were similar to estimates reported in prior studies of Asian ${ }^{14}$ and European ${ }^{13}$ populations; however, only rs9398652 reached genome-wide significance in the current meta-analysis. The rs9398652 SNP was approximately $30 \mathrm{~kb}$ downstream and in high linkage disequilibrium with the leading SNP from the present study (rs9320841; CEU $r^{2}=1.00$; YRI $r^{2}=.81$ ). In addition, rs 12110693 was in strong linkage disequilibrium with rs9320841 (rs9320841; CEU $r^{2}=1.00$; YRI $r^{2}=.76$ ) (Table 2). The final reported SNP from the 6q22 locus, rs11154022, did not reach genome-wide significance, was the greatest distance from rs9320841 (approximately $365 \mathrm{~kb}$ upstream), and not in LD with it (CEU $r^{2}=.01$; YRI $\left.r^{2}=.01\right)$.

Other variants that were identified from prior European and Asian GWAS were also tested (Table 2). The 2 SNPs that have previously been identified at the MYH6 locus (rs452036 and rs365990) were associated with resting heart rate in African Americans using the replication threshold $\left(3.85 \times 10^{-3}\right)$. These variants are associated with a similar increase in the sinus cycle length across Europeans, Asians, and African Americans. We were unable to confirm associations for several previously published loci at replication thresholds: $C D 46$ on chromosome 1, SLC35F1 on chromosome 6, SLC12A9 and UfSp1 on chromosome 7, FADS1 on chromosome 11, an intergenic region on chromosome 12, GPR133 on chromosome 12, and $M Y H 7$ on chromosome 14 (Table 2). These findings were consistent across the different cohorts analyzed through the CARe-COGENT consortium (Figure 5). Further evaluation of these loci (the $1 \mathrm{Mb}$ regions, $500 \mathrm{~kb}$ upstream and downstream of the SNPs in Table 2) did not identify any other genome-wide significant RR-SNP associations despite having adequate power (power $>0.8$ ).

\section{Discussion}

In a large GWAS of African Americans, we generalized a previously reported association between a variant on chromosome 6q22.31 and resting heart rate to a population of African descent. The present findings suggest that rs9320841, which is located in an intergenic region $350 \mathrm{~kb}$ downstream from $G J A 1$, is the leading SNP at this locus associated with heart rate. In addition, rs9320841 is in high LD with other intergenic SNPs from this region previously associated with heart rate in GWAS in populations of European and Asian ancestry. ${ }^{13,14}$

Multiple studies including the current report have demon strated intergenic SNPs in proximity to rs9320841 that are associated with variation in heart rate among individuals of Asian, European, and African ancestry. The closest putative transcript to rs9320841 on chromosome 6q22.31 is $H M G B 3 P 18$, which has no known function. However, GJA1 which is approximately $350 \mathrm{~kb}$ upstream of this SNP, encodes connexin 43, the main cardiac gap junction channel that is found throughout the heart and is responsible for intercellular con ductance in the atria and ventricles. ${ }^{27}$ Connexin 43 is expressed abundantly in the atria and permits the node to conduct impulses to the surrounding muscle. ${ }^{28}$ Experimental models have demon strated that the deletion of various gap junction subunits results in a sick sinus syndrome phenotype with bradycardia, sinus dysrhythmia, and sinus node exit block. ${ }^{29,30}$ 
As a result, these intergenic variants in the $6 \mathrm{q} 22$ locus, which are in close proximity to GJA1 and have been identified across different populations, may reduce sinus automaticity.

Although rs9320841 and previously identified 6q22.31 loci are 300-500 kb away from and in low LD with SNPs in GJA1 recent studies suggest that variations in intergenic regions may regulate transcription factor binding and chromatin modifica tion. ${ }^{31}$ Functional and translational studies focused on this intergenic region on chromosome $6 \mathrm{q} 22$ will be required to understand its potential effect on GJA1.

In the portion of our study that restricted the analysis to previously identified variants, we observed an association between 2 SNPs located within the $M Y H 6$ gene and resting heart rate. $M Y H 6$ encodes one of the myosin heavy chain subunits in the cardiac sarcomere and is a major component of the cardiac contractile system. In addition, $M Y H 6$ encodes a cardiacspecific microRNA, miR-208a, which is a key regulatory molecule that is necessary for normal cardiac conduction. ${ }^{32}$ Specifically, miR-208a regulates expression of connexin 40 , a gap junction protein that is implicated in sinus automaticity and cardiac arrhythmias. ${ }^{29-33}$ As a result, changes in the $M Y H 6$ genetic architecture could alter microRNA production, gap junction formation, and sinus node function. Prior GWAS in European populations have identified common variants in this gene to be associated with resting heart rate ${ }^{13,15}$ and rare variants, located $0.3-4.4 \mathrm{~kb}$ from these SNPs, to be associated with sick sinus syndrome. ${ }^{34}$ Although SNPs at the $M Y H 6$ locus were not identified in the discovery phase of our analysis at genome-wide significance thresholds $\left(P<2.5 \times 10^{-8}\right)$, the similar magnitude and direction of the point estimates in our analysis suggest that the $M Y H 6$ gene affects sinus node automaticity in diverse populations.

While we were unable to replicate associations for other previously published loci at a threshold level of $3.85 \times 10^{-3}(0.05 / 13)$, the similar magnitude and direction of the point estimates suggest consistency across ancestries. Specifically, SLC12A9 and UfSp1 on chromosome 7 and the $M Y H 7$ region on chromosome 14 had effects on heart rate similar to those described by prior studies. Compared to individuals of European ancestry, however, African Americans have greater genetic diversity, ${ }^{18}$ which may lower the frequency of a particular allele and subsequently reduce the statistical likelihood of detecting an effect on the RR interval. In addition, linkage disequilibrium is commonly lower in African Americans ${ }^{35}$ and subsequently reduces the likelihood that a common SNP is in linkage disequilibrium with a causal variant. Furthermore, these analyses were conducted in a population that was predominantly woman, middle-aged, and overweight. This demographic profile differs from that of prior studies and may have influenced the results.

A common limitation of meta-analyses is among-study phenotype heterogeneity; however, the current study followed similar electrocardiographic and clinical protocols when measuring heart rate and its correlates. In addition, the statistical assessment of heterogeneity did not suggest large variation in SNP effects across studies. Moreover, the random-effects meta-analysis of these effects was weighted for both their within- and among-study variation. Another limitation of GWAS is potential for population stratification, including confounding by ancestry. However, we attempted to minimize bias from population structure by excluding participants of non-African ancestry, adjusting for principal components in study-specific regression models, and applying genomic control methods.

\section{Conclusions}

In summary, the genome-wide significance of an association linking resting heart rate and the GJA1 locus previously described in European and Asian populations has now been 
generalized to African Americans. In addition, this analysis has replicated associations initially discovered in Europeans between common variants within the $M Y H 6$ gene and a reduction in heart rate to an African American population. Generalizability across global populations and biological plausibility of the heart rate- $G J A 1$ and heart rate-MYH6 associations highlight the potential importance of these loci in the intrinsic (nodal and myocardial) determination of resting heart rate.

\section{Supplementary Material}

Refer to Web version on PubMed Central for supplementary material.

\section{Acknowledgments}

Atherosclerosis Risk in Communities (ARIC): The ARIC study is carried out as a collaborative study supported by National Heart, Lung, and Blood Institute (NHLBI) contracts to the University of North Carolina at Chapel Hill (N01-HC-55015), Baylor Medical College (N01-HC-55016), University of Mississippi Medical Center (N01HC-55021), University of Minnesota (N01-HC-55019), Johns Hopkins University (N01-HC-55020), University of Texas, Houston (N01-HC-55022), and University of North Carolina, Forsyth County (N01-HC-55018). Baltimore Longitudinal Study of Aging (BLSA): The BLSA was supported in part by the Intramural Research Program of the National Institutes of Health (NIH), National Institute on Aging (NIA). A portion of that support was through an R\&D contract with MedStar Research Institute. Bogalusa Heart Study (BHS): Dr Smith, Dr Murray, and Dr Schork were supported in part by NIH/National Center for Research Resources (NCRR) grant number UL1 RR025774 and Scripps Genomic Medicine. The BHS was supported by grants HD-061437 and HD-062783 from the National Institute of Child Health and Human Development and AG-16592 from the NIA. Cleveland Family Study (CFS): This study was supported by grant to Case Western Reserve University (NIH HL 46380, M01RR00080). Cardiovascular Health Study (CHS): This CHS research was supported by NHLBI contracts N01-HC-85239, N01-HC-85079 through N01-HC-85086, N01-HC-35129, N01 HC-15103, N01 HC-55222, N01HC-75150, N01-HC-45133, and HHSN268201200036C and NHLBI grants HL080295, HL087652, HL105756, and HL085251 with additional contribution from National Institute of Neurological Disorders and Stroke (NINDS). Additional support was provided through AG-023629, AG-15928, AG-20098, and AG-027058 from the NIA. See also http://www.chs-nhlbi.org/pi.htm. DNA handling and genotyping were supported in part by National Center of Advancing Translational Technologies CTSI grant UL1TR000124 and National Institute of Diabetes and Digestive and Kidney Diseases grant DK063491 to the Southern California Diabetes Endocrinology Research Center. The Health, Aging, and Body Composition (Health ABC) study: The Health ABC study was supported by NIA contracts N01AG62101, N01AG62103, and N01AG62106. The genome-wide association study was funded by NIA grant 1R01AG032098-01A1 to Wake Forest University Health Sciences and genotyping services were provided by the Center for Inherited Disease Research (CIDR). CIDR is fully funded through a federal contract from the NIH to Johns Hopkins University (contract number HHSN268200782096C). This research was supported in part by the Intramural Research Program of the NIH, NIA. The Healthy Aging in Neighborhoods of Diversity across the Life Span (HANDLS) study: This HANDLS study was supported by the Intramural Research Program of the NIH, NIA, and the National Center on Minority Health and Health Disparities (contract number Z01AG000513 and human subjects protocol number 2009-149). Data analyses for the HANDLS study used the highperformance computational capabilities of the Biowulf Linux cluster at the NIH (http://biowulf.nih.gov). Jackson Heart Study (JHS): This JHS was supported by NIH contracts N01-HC-95170, N01-HC-95171, and N01HC-95172 provided by the NHLIB and the National Center for Minority Health and Health Disparities. MultiEthnic Study of Atherosclerosis (MESA): This study was supported by grants to the University of Washington (N01-HC-95159), Regents of the University of California (N01-HC-95160), Columbia University (N01HC-95161), Johns Hopkins University (N01-HC-95162, N01-HC-95168), University of Minnesota (N01HC95163), Northwestern University (N01-HC-95164), Wake Forest University (N01-HC-95165), University of Vermont (N01-HC-95166), New England Medical Center (N01-HC-95167), Harbor-UCLA Research and Education Institute (N01-HC-95169), Cedars-Sinai Medical Center (R01-HL-071205), and University of Virginia (subcontract to R01-HL-071205). Women's Health Initiative (WHI): The WHI program is funded by the NHLBI, NIH, US Department of Health and Human Services through contracts N01WH22110, 24152, 32100-2, 32105-6, 32108-9, 32111-13, 32115, 32118-32119, 32122, 42107-26, 42129-32, and 44221. This article was prepared in collaboration with the investigators of WHI and has been reviewed and/or approved by WHI. WHI investigators are listed at http://www.whiscience.org/publications/WHI_investigators_shortlist.pdf. Funding for WHI SHARe genotyping was provided by NHLBI contract N02-HL-64278. Analyses in WHI were funded by the NIH/NIEHS (1-R01-ES017794, Whitsel) and the NIH/NCI (N01-WH-2-2110, North). Dr Deo was supported by K23DK089118 from the NIH. Dr Avery was partially supported by NHLBI/NIH grant R00HL098458. Dr Smith was supported by the Swedish Heart-Lung Foundation. 


\section{ABBREVIATIONS}

ARIC
BHS
BLSA
CFS
CHS
CARe
COGENT
ECG
HANDLS
Health ABC
GWAS
JHS
MESA
SNP
WHI

Atherosclerosis Risk in Communities study

Bogalusa Heart Study

Baltimore Longitudinal Study on Aging

Cleveland Family Study

Cardiovascular Health Study

Candidate-gene Association Resource

Continental Origins and Genetic Epidemiology Network

electrocardiogram

Healthy Aging in Neighborhoods of Diversity across the Life Span Study

Health Aging and Body Composition

genome-wide association study

Jackson Heart Study

Multi-Ethnic Study of Atherosclerosis

single nucleotide polymorphism

Women's Health Initiative clinical trials

\section{References}

1. Greenland P, Daviglus ML, Dyer AR, et al. Resting heart rate is a risk factor for cardiovascular and noncardiovascular mortality: the Chicago Heart Association Detection Project in Industry. Am J Epidemiol. 1999; 149:853-862. [PubMed: 10221322]

2. Nauman J, Nilsen TI, Wisloff U, Vatten LJ. Combined effect of resting heart rate and physical activity on ischaemic heart disease: mortality follow-up in a population study (the HUNT study, Norway). J Epidemiol Community Health. 2010; 64:175-181. [PubMed: 20056969]

3. Kristal-Boneh E, Silber H, Harari G, Froom P. The association of resting heart rate with cardiovascular, cancer and all-cause mortality: eight year follow-up of 3527 male Israeli employees (the CORDIS Study). Eur Heart J. 2000; 21:116-124. [PubMed: 10637085]

4. Chang M, Havlik RJ, Corti MC, Chaves PH, Fried LP, Guralnik JM. Relation of heart rate at rest and mortality in the Women's Health and Aging Study. Am J Cardiol. 2003; 92:1294-1299. [PubMed: 14636906]

5. Jouven X, Empana JP, Escolano S, et al. Relation of heart rate at rest and long-term (>20 years) death rate in initially healthy middle-aged men. Am J Cardiol. 2009; 103:279-283. [PubMed: 19121452]

6. Jouven X, Zureik M, Desnos M, Guerot C, Ducimetiere P. Resting heart rate as a predictive risk factor for sudden death in middle-aged men. Cardiovasc Res. 2001; 50:373-378. [PubMed: 11334841]

7. Fox K, Borer JS, Camm AJ, et al. Resting heart rate in cardiovascular disease. J Am Coll Cardiol. 2007; 50:823-830. [PubMed: 17719466]

8. Osztovits J, Horvath T, Littvay L, et al. Effects of genetic vs. environmental factors on cardiovascular autonomic function: a twin study. Diabet Med. 2011; 28:1241-1248. [PubMed: 21679234]

9. Russell MW, Law I, Sholinsky P, Fabsitz RR. Heritability of ECG measurements in adult male twins. J Electrocardiol. 1998; 30:64-68. [PubMed: 9535482]

10. Singh JP, Larson MG, O’Donnell CJ, Tsuji H, Evans JC, Levy D. Heritability of heart rate variability: the Framingham Heart Study. Circulation. 1999; 99:2251-2254. [PubMed: 10226089] 
11. Martin LJ, Comuzzie AG, Sonnenberg GE, et al. Major quantitative trait locus for resting heart rate maps to a region on chromosome 4. Hypertension. 2004; 43:1146-1151. [PubMed: 14993199]

12. Marroni F, Pfeufer A, Aulchenko YS, et al. A genome-wide association scan of RR and QT interval duration in 3 European genetically isolated populations: the EUROSPAN project. Circ Cardiovasc Genet. 2009; 2:322-328. [PubMed: 20031603]

13. Eijgelsheim M, Newton-Cheh C, Sotoodehnia N, et al. Genome-wide association analysis identifies multiple loci related to resting heart rate. Hum Mol Genet. 2010; 19:3885-3894. [PubMed: 20639392]

14. Cho YS, Go MJ, Kim YJ, et al. A large-scale genome-wide association study of Asian populations uncovers genetic factors influencing eight quantitative traits. Nat Genet. 2009; 41:527-534. [PubMed: 19396169]

15. Holm H, Gudbjartsson DF, Arnar DO, et al. Several common variants modulate heart rate, PR interval and QRS duration. Nat Genet. 2010; 42:117-122. [PubMed: 20062063]

16. A haplotype map of the human genome. Nature. 2005; 437:1299-1320. [PubMed: 16255080]

17. Tishkoff SA, Williams SM. Genetic analysis of African populations: human evolution and complex disease. Nat Rev Genet. 2002; 3:611-621. [PubMed: 12154384]

18. Tennessen JA, Bigham AW, O'Connor TD, et al. Evolution and functional impact of rare coding variation from deep sequencing of human exomes. Science. 2012; 337:64-69. [PubMed: 22604720]

19. Musunuru K, Lettre G, Young T, et al. Candidate gene association resource (CARe): design, methods, and proof of concept. Circ Cardiovasc Genet. 2010; 3:267-275. [PubMed: 20400780]

20. Reiner AP, Lettre G, Nalls MA, et al. Genome-wide association study of white blood cell count in 16,388 African Americans: the continental origins and genetic epidemiology network (COGENT). PLoS Genet. 2011; 7:e1002108. [PubMed: 21738479]

21. The CSE Working Party. Recommendations for measurement standards in quantitative electrocardiography. Eur Heart J. 1985; 6:815-825. [PubMed: 4076195]

22. Price AL, Patterson NJ, Plenge RM, Weinblatt ME, Shadick NA, Reich D. Principal components analysis corrects for stratification in genome-wide association studies. Nat Genet. 2006; 38:904909. [PubMed: 16862161]

23. Li Y, Willer C, Sanna S, Abecasis G. Genotype imputation. Annu Rev Genom Hum Genet. 2009; 10:387-406.

24. Chen MH, Yang Q. GWAF: an R package for genome-wide association analyses with family data. Bioinformatics. 2010; 26:580-581. [PubMed: 20040588]

25. Devlin B, Roeder K. Genomic control for association studies. Biometrics. 1999; 55:997-1004. [PubMed: 11315092]

26. Pe'er I, Yelensky R, Altshuler D, Daly MJ. Estimation of the multiple testing burden for genomewide association studies of nearly all common variants. Genet Epidemiol. 2008; 32:381385. [PubMed: 18348202]

27. Gros DB, Jongsma HJ. Connexins in mammalian heart function. Bioessays. 1996; 18:719-730. [PubMed: 8831288]

28. Boyett MR, Inada S, Yoo S, et al. Connexins in the sinoatrial and atrioventricular nodes. Adv Cardiol. 2006; 42:175-197. [PubMed: 16646591]

29. Hagendorff A, Schumacher B, Kirchhoff S, Luderitz B, Willecke K. Conduction disturbances and increased atrial vulnerability in connexin40-deficient mice analyzed by transesophageal stimulation. Circulation. 1999; 99:1508-1515. [PubMed: 10086977]

30. Dobrzynski H, Boyett MR, Anderson RH. New insights into pacemaker activity: promoting understanding of sick sinus syndrome. Circulation. 2007; 115:1921-1932. [PubMed: 17420362]

31. Harismendy O, Notani D, Song X, et al. 9p21 DNA variants associated with coronary artery disease impair interferon-gamma signalling response. Nature. 2011; 470:264-268. [PubMed: 21307941]

32. Callis TE, Pandya K, Seok HY, et al. Microrna-208a is a regulator of cardiac hypertrophy and conduction in mice. J Clin Invest. 2009; 119:2772-2786. [PubMed: 19726871] 
33. Simon AM, Goodenough DA, Paul DL. Mice lacking connexin40 have cardiac conduction abnormalities characteristic of atrioventricular block and bundle branch block. Curr Biol. 1998; 8:295-298. [PubMed: 9501069]

34. Holm H, Gudbjartsson DF, Sulem P, et al. A rare variant in MYH6 is associated with high risk of sick sinus syndrome. Nat Genet. 2011; 43:316-320. [PubMed: 21378987]

35. Rosenberg NA, Huang L, Jewett EM, Szpiech ZA, Jankovic I, Boehnke M. Genome-wide association studies in diverse populations. Nat Rev Genet. 2010; 11:356-366. [PubMed: 20395969]

\section{Appendix A Supplementary data}

Supplementary data associated with this article can be found in the online version at http:// dx.doi.org/10.1016/j.hrthm.2012.11.014. 

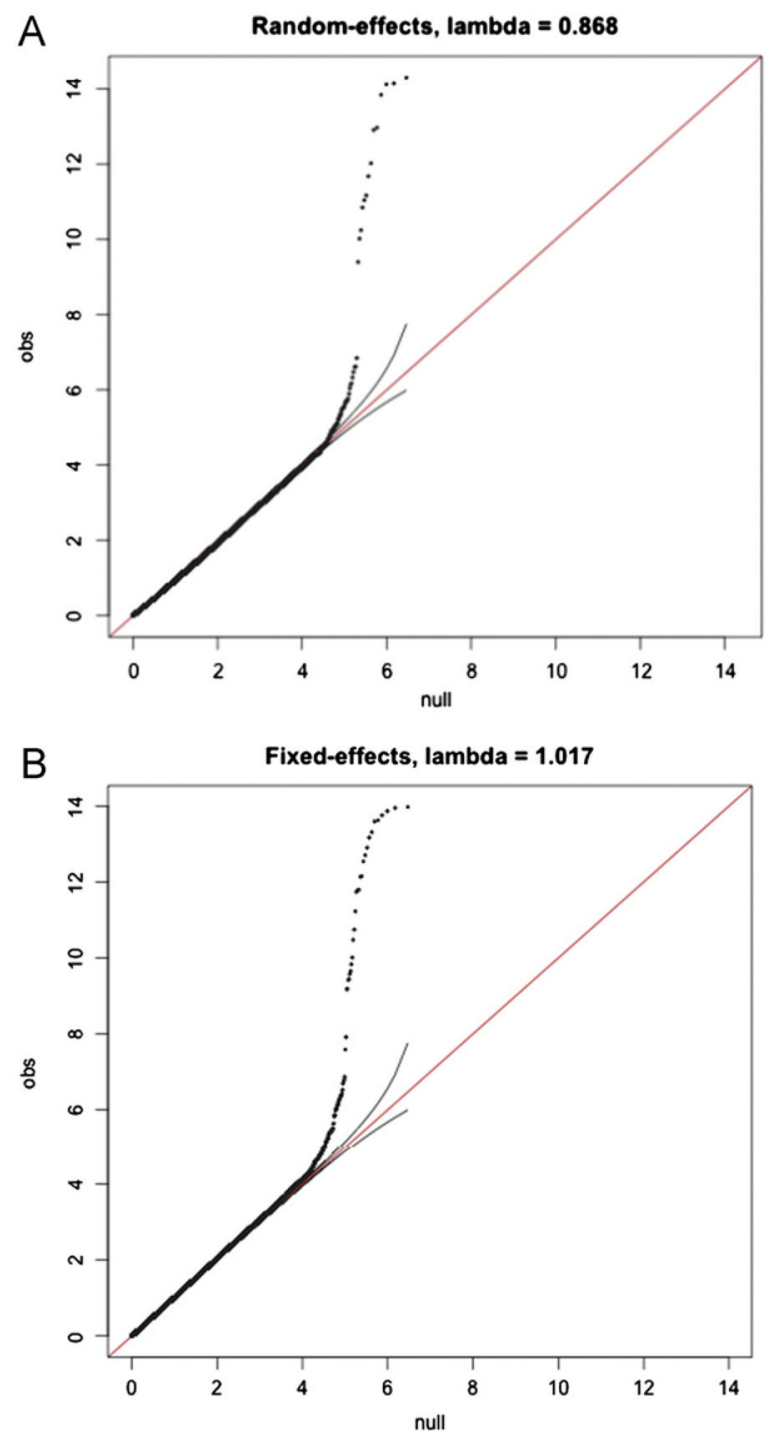

Figure 1.

QQ plots of meta-analysis using either random-effects (A) or fixed-effects (B) modeling. The $\mathrm{x}$ axis marks the expected values, and the left-hand $\mathrm{y}$ axis marks the observed values. A line originating from the origin and having a slope of 1 is depicted in red. 


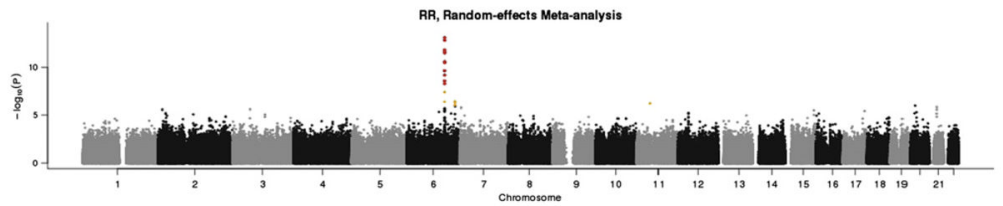

Figure 2.

Manhattan plot of RR associations for all SNPs. The $P$ values from random-effects metaanalysis of 2,954,965 successfully imputed or genotyped SNPs in $\geq 2$ cohorts. Red points $=$ SNPs with $P<2.5 \times 10^{-8}$ (considered genome-wide significant). Orange points $=$ SNPs with $P$ values ranging from less than $1 \times 10^{-5}$ to $2.5 \times 10^{-8}$. Regions containing red points were considered genome-wide significant. SNP = single nucleotide polymorphism. 


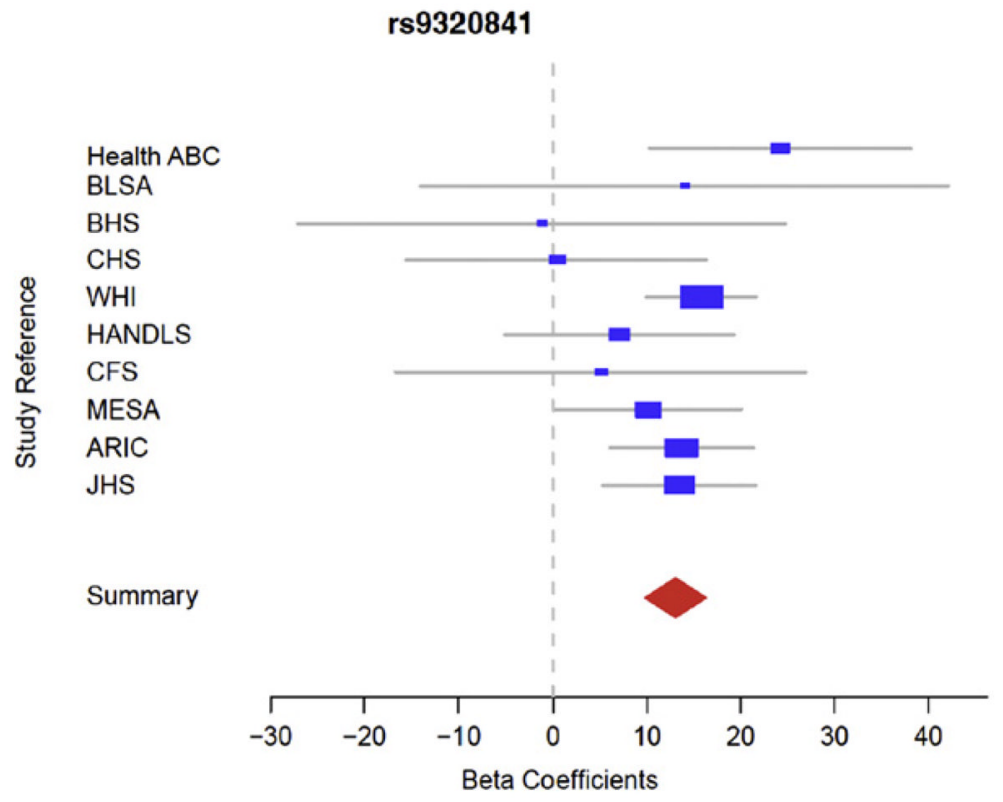

Figure 3.

Forest plot depicting the effect (beta coefficient) of rs9320841 on RR in milliseconds per allele (95\% confidence interval) across the individual cohort studies and overall using random-effects modeling $\left(\mathrm{I}^{2}=0\right)$. ARIC $=$ Atherosclerosis Risk in Communities study; BHS $=$ Bogalusa Heart Study; BLSA $=$ Baltimore Longitudinal Study on Aging; CFS = Cleveland Family Study; CHS = Cardiovascular Health Study; HANDLS = Healthy Aging in Neighborhoods of Diversity across the Life Span Study; Health ABC = Health Aging and Body Composition; JHS = Jackson Heart Study; MESA = Multi-Ethnic Study of Atherosclerosis; WHI = Women's Health Initiative clinical trials. 


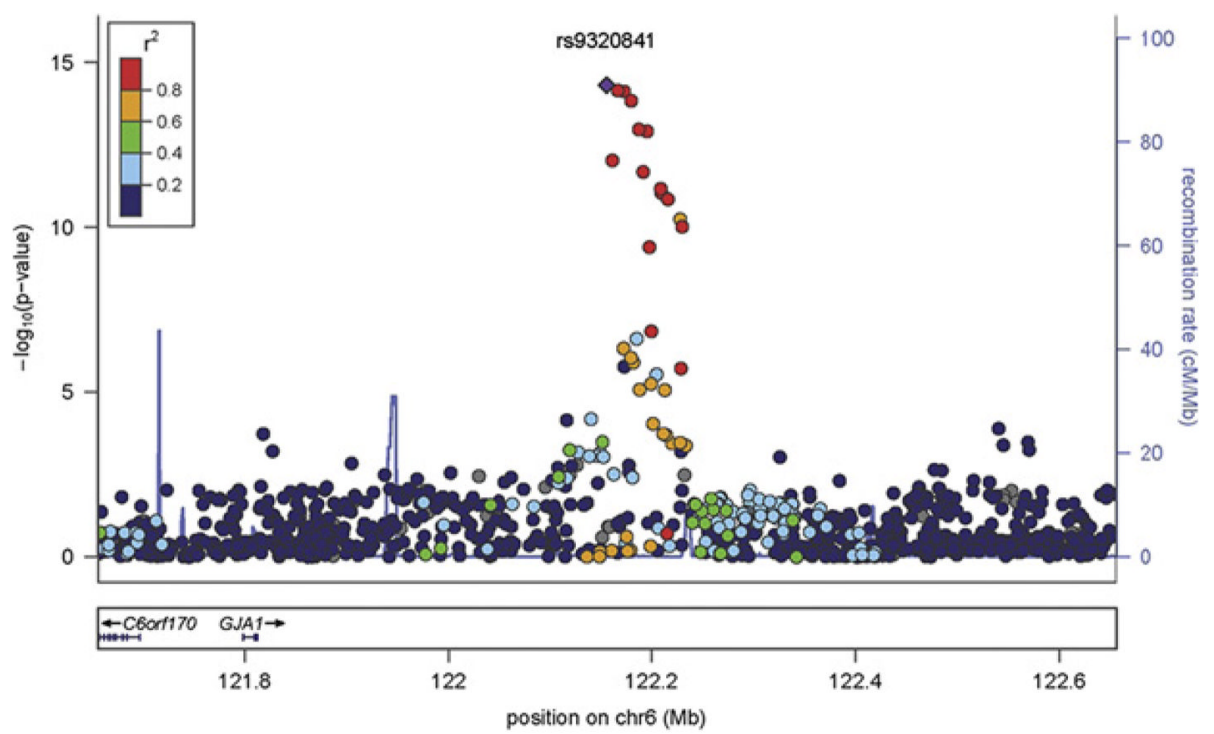

Figure 4.

Regional association plots for the RR interval plotted using $P$ values estimated from 13,372 African Americans from 10 studies. Positions are from NCBI build 36. Linkage disequilibrium and recombination rates are estimated from HapMap phase II data. SNPs are represented by circles. The large blue diamond is the SNP with the lowest $P$ value. The circle color represents correlation with the top SNP: blue indicates weak correlation, and red indicates strong correlation. Recombination rate is plotted in the background, and known genes in the region are shown at the bottom of the plot. SNP $=$ single nucleotide polymorphism. 

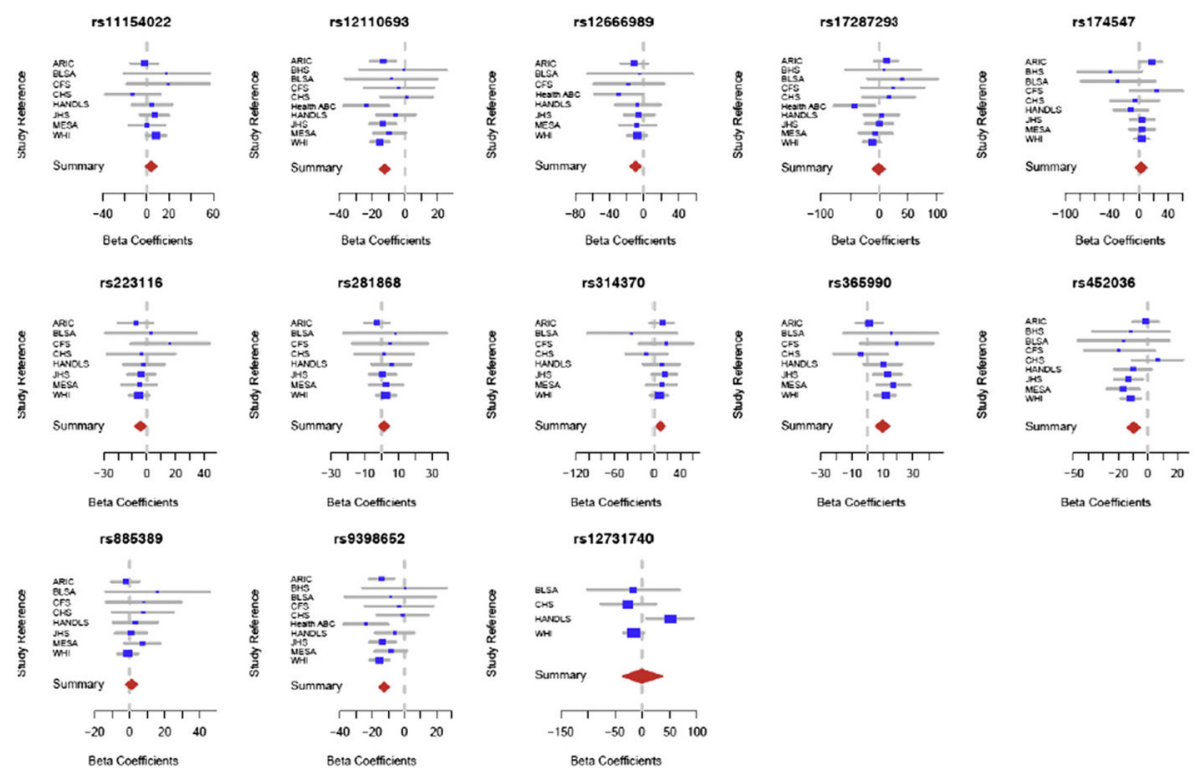

Figure 5.

Forest plot depicting the effect in milliseconds per allele of SNPs achieving genome-wide significance in European and Asian studies across the individual African American cohorts. $\mathrm{SNP}=$ single nucleotide polymorphism. 


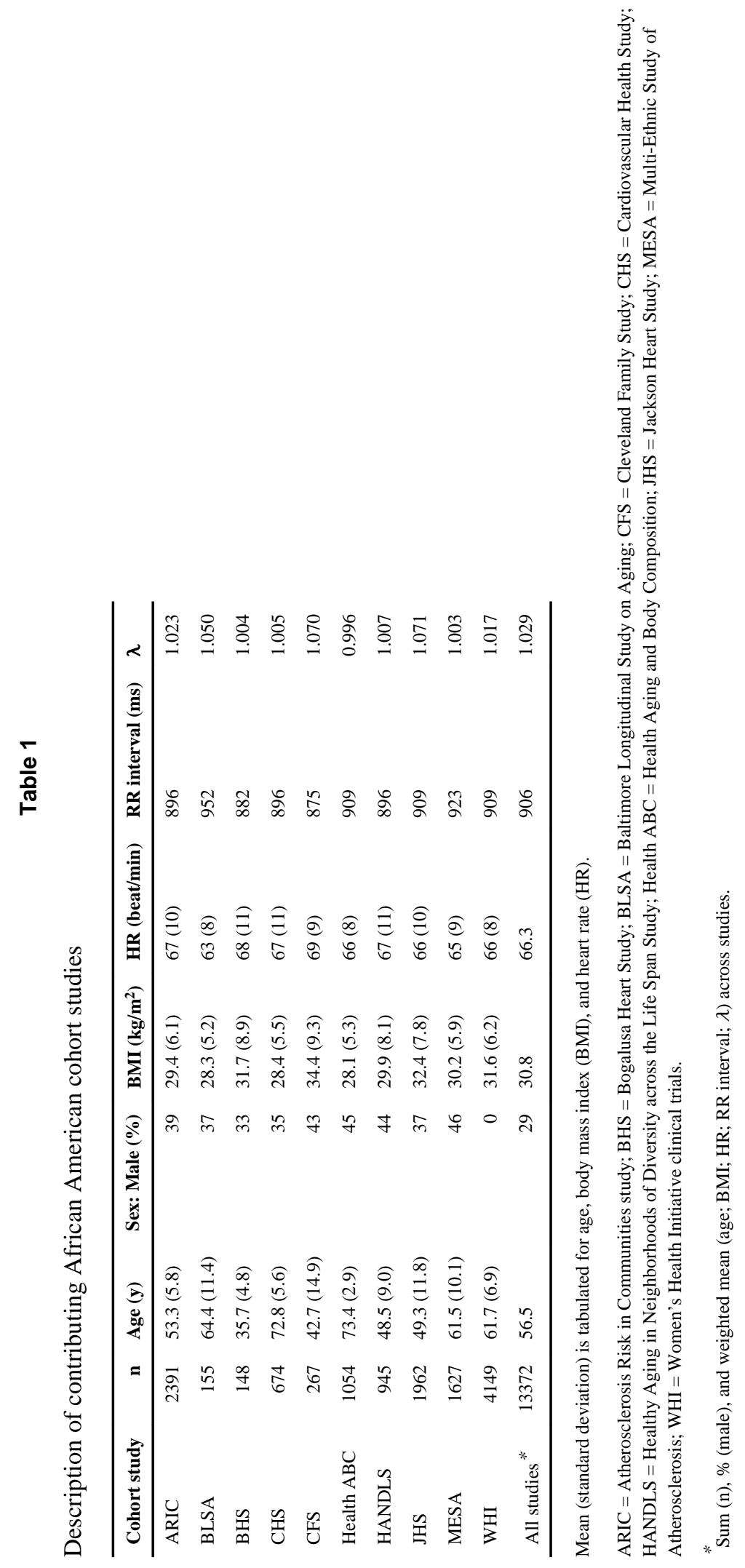

Heart Rhythm. Author manuscript; available in PMC 2014 March 01. 
